\title{
Critical Systematic Errors in the Implementation and Follow-up of Performance in the Hematology Area: A Prospective Study
}

\author{
Jeel Moya-Salazar ${ }^{1,2}$ and Liz Pio-Dávila ${ }^{3}$ \\ ${ }^{1}$ Departamento Académico de Microbiología Médica, Universidad Nacional Mayor de San Marcos; ${ }^{2}$ Hospital Nacional Docente Madre Niño San \\ Bartolomé; ${ }^{3}$ Laboratorio de Análisis Clínicos, Clínica Universitaria. Lima, Peru
}

\begin{abstract}
Due to the low use of the quality assessment of the hematological laboratories under the critical systematic error ( $\triangle S E$ ), and with the imperative to explore the implications of the use of the average of standard deviations and the control charts of each hematological parameter, we developed a prospective research at the University Clinic in Lima, Peru. We analyzed eight hematological parameters under the CLSI EP-15 A2 and CLSI H26-A2 guidelines for 19 days in the Landwind LW D3600 hematology autoanalyzer. In total, 1368 assays were performed (171 runs for each hematological parameter and 57 for each control level), which generated 19 averages in each level and for each parameter where we found 90 violations of the Westgard rules. Performance characteristics were optimal for leukocyte count (white blood cell) and platelets $(\Delta S E=\geq 5.8$ ) and were deficient for all erythrocyte parameters $(\triangle S E=0.17-3.27)$. We identify significant differences between the determined automatic mean (by the autoanalyzer) and the calculated mean, having in many cases marked the difference. The quality evaluation of the $\triangle S E$ analysis showed a heterogeneous performance and intra-parameter control levels, as well as violations of the Westgard rules and mismatches in the determination of means (calculated and automatic).
\end{abstract}

Key words: Quality control. Hematology. Critical systematic error. Average. Primary health care level.

\section{Introduction}

In recent years, new tools for the diagnosis of different diseases have been created (i.e., cancer and malaria and among others). In the pursuit of assuring quality results, reducing diagnostic costs, and improving their performance, while making them more accessible to different sanitary realities ${ }^{1-5}$.

This development needs a quality guarantee which ensures trustworthy, efficient, and sustainable results, with a low error rate within the health-care systems. An inadequate quality in health-care endangers the patients' health. By 1999, estimations in the US suggested that approximately 98,000 deaths were caused by medical errors ${ }^{6}$.

In clinical laboratories, quality is an ethical requirement and an imperative dynamic practice, which will allow us to issue results closer to the physiological reality of the patients. This assurance of quality is the initial component to ensure each stage of the diagnostic process $^{7}$. From verification up to quality control, there are guidelines, parameters, and recommendations which assist clinical laboratories in improving processes and establishing optimal solutions ${ }^{8,9}$.

In hematologic laboratories, performance Six sigma evaluations of performance and quality are rare. The

\section{Correspondence:}

Jeel Moya-Salazar

E-mail: jeel.moyasalazar@icloud.com
Date of reception: 31-10-2017

Date of acceptance: 16-01-2018

DOI: 10.24875/RMU.M18000010
Available online: 30-05-2018 Medicina Universitaria. 2018;20(1):22-34 www.medicinauniversitaria.org

-5796/C 2018 Universidad Autónoma de Nuevo Leon. Published by Permanyer México SA de CV. This is an open access article under the CC BY-NC-ND license (http://creativecommons.org/licenses/by-nc-nd/4.0/). 
most significant performance evaluation process of, for example, hematologic auto-analyzers is based on the fact that the standard deviations (SD) of hematologic parameters do not exceed the limits established by the maker, and that there are no errors shown in daily control graphics reflected in some infraction of the Westgard rules ${ }^{10-15}$. In 1963, Dorsey described the use of a daily mean of "mean corpuscular hemoglobin $(\mathrm{MCH})$," this fact almost went unnoticed up until the development of the Bull algorithm for erythrocyte indexes (red blood cell [RBC]) in 1974, representing the first quality control system for hematologic counters ${ }^{16}$. The benefits of quality management systems in different countries and health centers are unknown ${ }^{17,18}$.

The objective of this investigation was to develop a quality evaluation of the critical systematic error $(\Delta S E)$ in the implementation and follow-up of hematologic performance in Lima, Peru, against the analysis comparative means of 8 hematologic parameters.

\section{Materials and methods}

To accomplish the established objectives, a prospective investigation was conducted at the Clinical Analysis Laboratory at the University Clinic in Lima, Peru. This study was approved by all institutions involved in its completion.

\section{Automated analyzer}

Conducive to performing determinations, an open-system Landwind Auto hematology analyzer LW D3600 was utilized (Landwind Medical Co., Shenzhen, China) for the analysis of 3 differentials, 20 parameters, and three histograms. This system processes $9.6 \mu \mathrm{l}$ of venous or arterial blood through an analytic run ${ }^{11}$. For this study, the equipment was not switched off and was stabilized $>30$ min before each daily use.

\section{Reactives}

Diagnostic reagents from COMING, Sozhou, China, were used (diluent reactives for hematology analyzer [Lot 2016121001], Lyser for hematology analyzer [Lot 20160707], and Cleaner for hematology analyzer [Lot 2016121601]). Hematologic controls were open-system reactives of three control levels (low, normal, and high) Myt-3D MYTHIC 18 (Lot B0816) from Orpée SA, Geneva, Switzerland. All analyses were conducted following the institution's operative necessities (Standardized
Operational Procedure or SOP), according to the maker's recommendations within the evaluation system.

\section{Hematologic parameters}

Analysis parameters were the leukocytes count (white blood cell [WBC]) $\times 10^{3} / \mu \mathrm{L}, \mathrm{RBC} \times 10^{3} / \mu \mathrm{L}$, dosage of hemoglobin (HB) in $\mathrm{g} / \mathrm{dL}$, calculated automated hematocrit (HCT) in percentage. Furthermore, mean corpuscular volume (MCV) in $\mathrm{fL}, \mathrm{MCH}$ in $\mathrm{pL}$, mean corpuscular hemoglobin concentration $(\mathrm{MCHC})$ in $\mathrm{g} / \mathrm{dL}$, and platelet count (PLT) $\times 10^{3} / \mu \mathrm{L}$.

\section{Quality evaluation}

The quality process was based on the evaluation of selected parameters during 19 days under the requirement guides CLSI EP-15 A2 and CLSI H26-A2 $2^{19,20 .}$ Before the beginning of the study, the system was calibrated under the maker's guidelines and an external quality control (data not shown). During the time of the study, control runs were conducted at the beginning of the work shift ( $12 \mathrm{~h}$ ) 30 times before mixing the analysis tube $^{21}$. Each control level (low, normal, and high) was run in triplicate, obtaining an average value calculated by the auto hematology analyzer's software, which was established as a daily control value. No repetitions of controls were performed.

To evaluate whether or not the Landwind LW D3600 data system reaches quality specifications, the $\Delta S E$ was calculated based on the Six sigma value $(\Delta S E=\sigma-1.65)$ having previously obtained the quality bias, inaccuracy, and requirement values (TEa) for each hematologic parameter ${ }^{20}$. The results of this evaluation were drawn on power function graphs indicating the quality control rules ${ }^{10}$.

\section{Data analysis}

Under the CLSI EP15-A2 guidelines, the mean SD or variation coefficient of each hematologic parameter was obtained, taking into consideration CLSI quality requirements on biologic variability for $\mathrm{RBC}, \mathrm{WBC}, \mathrm{PLT}$, $\mathrm{HB}, \mathrm{MCV}, \mathrm{HCT}$, and Rico's EQA values for MCH and $\mathrm{MCHC}^{20,22}$. Based on the auto hematology analyzer's automatic average, they were compared with the calculations of the manual means (calculated average), which were compared through a simple linear regression. All runs were drawn on a chart based on SD for each parameter and were correlated with the sigma and $\triangle$ SE values using the Pearson correlation. The 
data analysis was conducted using the IBM SPSS v21 statistical analyzer (Armonk, US) and MS-Excel 2010 (Redmond, US) for Windows.

\section{Results}

A total of 1368 runs were conducted (171 runs for each hematologic parameter and 57 at each control level), which generated 19 averages at each level and for each parameter. Ranges and values expected for the analysis of hematologic parameters are shown in Table 1.

In Figures 1-3, we can see the quality evaluations of all hematologic parameters. From this analysis, agreements between the analyzed values and those established as standards were obtained. For RBC at the low level, there was a agreement in 15 analyses; at the normal level, we obtained 14 agreements, and at the high level there were 12 agreements. In the case of WBC, we obtained 36,18 , and 7 agreements at the low, normal, and high levels, respectively. Moreover, for PLT, we obtained 19 coincidences at the low level, 38 at the normal level, and 47 at the high level. There were also agreements for hematologic parameters. For HB, we obtained 21 agreements at the high level, 11 at the medium level, and only 3 at the low level. For HCT, we obtained 10 agreements at the high level, 14 at the normal level, and 15 at the low level.

In the erythrocytes indexes, MCV presented 107 agreements with the mean, 13 at the low level, 53 at the medium level, and 41 at the high level, followed by $\mathrm{MCH}$ with 61 agreements, 41 at the high level, 19 at the medium level, and one at the low level. MCHC had 20 agreements, 11 at the high level, and 9 at the medium level $(p=0.0001)$.

Exceeding the quality limits (SD) of each parameter resulted in an infraction of the main Westgard control rules (in 90 analyses). Unfortunately, following the institution's SOP no corrective actions were taken (control actions for these errors), and the samples were analyzed despite this rule infraction. Therefore, RBC's low control level showed 4 infractions of the rules $\left(1_{3 s}\right.$, $1_{2 s}, 6_{x}$, and $31_{s}$ ) at the normal and high levels (both with 2 infractions each); $2_{2 s}$, y $3_{1 s}$ were observed. For WBC, 15 infractions of the Westgard rules were observed, 5 at the low level $\left(1_{3 s}, 1_{2 s}, 9_{x}\right.$, and $\left.3_{1 s}\right), 5$ at the normal level $\left(1_{2 s}, 1_{2 x}\right.$, and $\left.3_{1 s}\right)$, and 4 at the high level $\left(2_{2 s}, 8_{x}\right.$ y $7_{T}$ ). In addition, the multilevel $2{ }_{\text {of }} 3_{2 s}$ infraction was observed (yellow circles - Fig. 1B). Furthermore, global infractions were also observed with PLT 12 (low level $1_{2 s}$ and $\left.9_{x}\right]$, normal level $1_{3 s}, 6_{x}$, and $9_{x}$ ], and high level $1_{2 \mathrm{~s}}$ and $\left.6_{\mathrm{x}}\right]$ ).
Likewise, for $\mathrm{HB}$, we observed 12 infractions of the Westgard rules: 6 at the low level $\left(3_{1 s}, 6_{x}, 9_{x}\right.$, and $\left.12_{x}\right)$, 2 at the normal level $\left(2_{2 s}\right.$ and $\left.12_{x}\right)$, and 2 at the high level $\left(1_{2 s}\right.$ and $\left.4_{1 s}\right)$. Furthermore, 2 multilevel infractions were observed $2_{\text {of }} 3_{2 s}$ and $3_{1 \mathrm{~s}}$ (light blue and yellow circles, respectively, - both in Fig. 2). For HCT, we observed a total of 10 infractions: 4 at the low level $\left(1_{2 s}\right.$, $6_{\mathrm{x}}$, and $\left.9_{\mathrm{x}}\right), 4$ at the normal level $\left(1_{2 \mathrm{~s}}, 3_{1 \mathrm{~s}}, 6_{\mathrm{x}}\right.$, and $\left.12_{\mathrm{x}}\right)$, and 2 multilevel infractions $3_{1} s$ (both yellow circles - Fig. 2B).

For hematimetric values, infractions were 9, 14, and 17 for MCV, MCH, and MHCMC, respectively. Two infractions were observed at the low level of MCV $\left(2_{2 s}\right.$ and $\left.9_{\mathrm{x}}\right), 3$ at the normal level $\left(2_{2 \mathrm{~s}}, 4_{1 \mathrm{~s}}\right.$, and $\left.9_{\mathrm{x}}\right)$, and 4 at the high level $\left(1_{2 s}, 2_{2 s}, 4_{1 s}\right.$, and $\left.9_{x}\right)$. In the $\mathrm{MCH}$ evaluation, we found 5 infractions at the low level $\left(1_{2 s}, 1_{3 s}\right.$, and $\left.6_{x}\right), 5$ infractions at the normal level $\left(1_{2 s}, 8_{x}, 9_{x}\right.$, and $\left.10_{x}\right), 3$ at the high level $\left(1_{2 s}, 1_{3 s}\right.$, and $\left.8_{x}\right)$, and a multilevel $3_{1 \mathrm{~s}}$ infraction (yellow circles - Fig. 3B). Finally, for MCHC, we found 6 infractions at the low level $\left(\mathcal{1}_{2 s}, 4_{1 \mathrm{~s}}\right.$, and $\left.10_{x}\right), 6$ infractions at the normal level $\left(1_{2 s}, 4_{1 s}, 10_{x}\right.$, and $\left.12_{x}\right)$, and 4 at the high level $\left(1_{2 s}, 4_{1 s}\right.$, and $\left.6_{x}\right)$, and a multilevel $3_{1 \mathrm{~s}}$ infraction (Yellow circles - Fig. $3 \mathrm{C}$ ).

The results of the analysis of the sigma metric and $\Delta S E$ are shown in Table 2. The hematological parameters that obtained a great performance were $\mathrm{WBC}$ and PLT, all the hematimetric indices $\mathrm{MCH}, \mathrm{MCHC}$, and $\mathrm{MCV}$ ) show a moderate/acceptable performance, and the values with low performance (poor quality) were RBC, HB, and HCT. A variability between control levels was observed within a parameter $(p=0.001)$ and between parameters $(p=0.022)$. Figure 4 shows example of the use of power charts for statistical control through $\Delta S E$.

Finally, from our correlation analysis of averages, we observed that at the low level, only RBC and HCT presented agreement in all tests among all methods of media analysis (calculated and automatic - Fig. 5). At this level of control, HB did not present agreement between their means. WBC, PLT, MCH, MCV, and $\mathrm{MCHC}$ had 3, 11, 4, 9, and 1 agreement, respectively. In the normal level, we observed 25 agreements of means, with HCT showing agreement in 18 of its 19 media, and RBC showing 13 agreements $(p=0.001)$. At this level, all the parameters present agreement in at least one analysis.

Finally, the results of the high level show that HCT shows agreement in all its means, followed by RBC. A contrario sense, the $\mathrm{MCH}$ did not observe agreement in any of their daily analyses. We also observed that other hematological parameters presented 1 (WBC), 2 
Table 1. Standard ranges and values for the analysis of hematological parameters Myt-3D MYTHIC 18 (Orpée SA, Geneva, Switzerland) of lot B0816 for 18 hematological parameters

\begin{tabular}{|l|c|c|c|c|c|c|c|c|}
\hline Controls (lot) & $\begin{array}{c}\text { WBC } \\
\left(\times 10^{3} / \mathbf{\mu L}\right)\end{array}$ & $\begin{array}{c}\mathbf{R B C} \\
\left(\times 10^{6} / \mathbf{\mu L}\right)\end{array}$ & $\begin{array}{c}\text { HB } \\
(\mathbf{g} / \mathbf{d L})\end{array}$ & $\begin{array}{c}\text { HCT } \\
(\%)\end{array}$ & $\begin{array}{c}\text { MCV } \\
(\mathbf{f L})\end{array}$ & $\begin{array}{c}\text { MCH } \\
(\mathbf{p g})\end{array}$ & $\begin{array}{c}\text { MCHC } \\
(\mathbf{g} / \mathbf{d L})\end{array}$ & $\begin{array}{c}\text { PLT } \\
\left(\times 10^{3} / \mathbf{\mu L}\right)\end{array}$ \\
\hline $\begin{array}{l}\text { Low } \\
\text { LOTB0816L }\end{array}$ & $2.0 \pm 0.5$ & $2.31 \pm 0.18$ & $62 \pm 4$ & $17.6 \pm 1.5$ & $76.0 \pm 5.0$ & $26.8 \pm 2.5$ & $353 \pm 3$ & $70 \pm 20$ \\
\hline $\begin{array}{l}\text { Normal } \\
\text { LOTB0816N }\end{array}$ & $7.2 \pm 1.0$ & $4.50 \pm 0.24$ & $134 \pm 6$ & $18.0 \pm 0.8$ & $83.0 \pm 5.0$ & $29.8 \pm 2.5$ & $356 \pm 3$ & $250 \pm 40$ \\
\hline $\begin{array}{l}\text { High } \\
\text { LOTB0816H }\end{array}$ & $19.7 \pm 2.5$ & $5.52 \pm 0.30$ & $180 \pm 8$ & $50.2 \pm 2.4$ & $91.0 \pm 5.0$ & $32.6 \pm 2.5$ & $358 \pm 3$ & $502 \pm 60$ \\
\hline
\end{tabular}

WBC: white blood cell, RBC: red blood cell, HB: hemoglobin, HCT: hematocrit, MCV: mean corpuscular volume, MCH: mean corpuscular hemoglobin, MCHC: mean corpuscular hemoglobin concentration, PLT: platelet

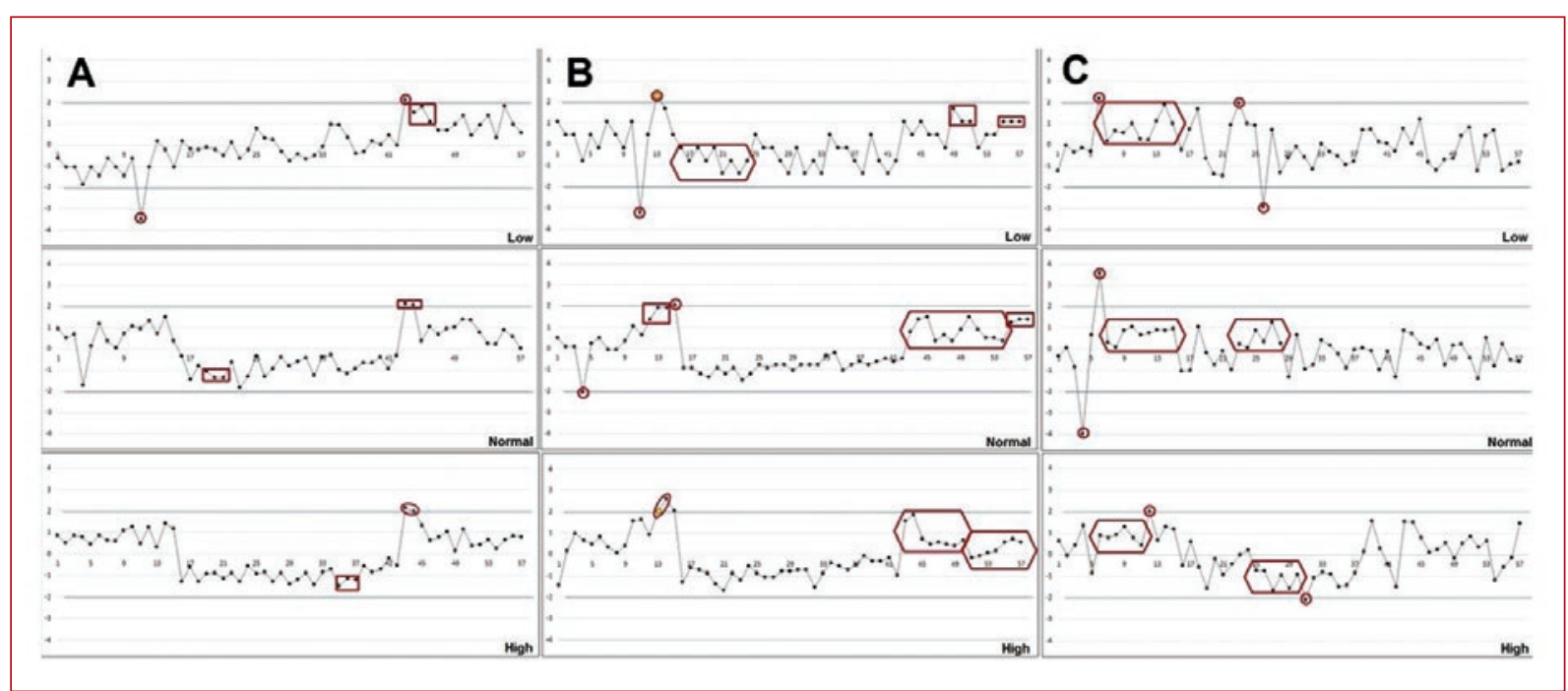

Figure 1. Levey-Jennings control chart of the daily runs of blood controls. The main infractions of the Westgard rules have been noted (red circles and squares). (A) Red blood cell $\left(\times 10^{6} / \mu \mathrm{L}\right)$, (B) white blood cell $\left(\times 10^{3} / \mu \mathrm{L}\right)$, (C) platelet $\left(\times 10^{3} / \mu \mathrm{L}\right)$. Lot: LOTB0816.

(HB and MCV), and 4 (PLT) agreements during the quality evaluation $(p<0.05)$.

\section{Discussion}

Developing a quality evaluation system under the $\triangle \mathrm{SE}$ analysis showed a heterogeneous performance between the hematological parameters and the intra-parameter control levels. Likewise, as a reflection of the high imprecision of each analysis, multiple infractions were observed at all levels of control, and significant differences were shown between the values of the automatic mean and calculated for most hematological parameters.

Clinical laboratories carry out tests with commercial hematological controls to demonstrate, as a final indicator, if what is determined in the analyzer (daily) is real. In other words, if each process is under control and offers results that are closest to the physiological reality of each patient. Quality assessment provides a unique opportunity to know "what is going wrong" and recognize the multiple factors involved in these processes, and to modify them, adapt them to working conditions to avoid medically important wrong results, and work within the framework of continuous improvement. 
Table 2. Summary of components of the quality evaluation under the analysis of critical systematic error $(\Delta \mathrm{SE})$ and sigma metric of eight hematological parameters

\begin{tabular}{|c|c|c|c|c|c|c|c|}
\hline Analysis & Level* & Average & Bias\% & CV\% & Tea** & Sigma & $\Delta$ SE \\
\hline \multirow[t]{3}{*}{ WBC $\left(\times 10^{3} / \mu \mathrm{L}\right)$} & Low & 2.26 & 0.25 & 10.97 & 7.5 & 7.5 & 5.83 \\
\hline & Medium & 4.39 & 0.30 & 6.75 & & 7.5 & 5.81 \\
\hline & High & 5.35 & 0.43 & 8.10 & & 7.4 & 5.80 \\
\hline \multirow[t]{3}{*}{$\mathrm{RBC}\left(\times 10^{6} / \mu \mathrm{L}\right)$} & Low & 1.93 & 0.16 & 8.41 & 3.0 & 3.0 & 1.33 \\
\hline & Medium & 7.04 & 0.71 & 10.05 & & 2.9 & 1.28 \\
\hline & High & 17.00 & 1.24 & 7.27 & & 2.8 & 1.18 \\
\hline \multirow[t]{3}{*}{$\mathrm{HB}(\mathrm{g} / \mathrm{dL})$} & Low & 53.46 & 5.52 & 10.32 & 3.5 & 3.0 & 1.32 \\
\hline & Medium & 121.49 & 11.89 & 9.79 & & 2.3 & 0.64 \\
\hline & High & 168.28 & 16.21 & 9.63 & & 1.8 & 0.17 \\
\hline \multirow[t]{3}{*}{ PLT $\left(\times 10^{3} / \mu \mathrm{L}\right)$} & Low & 114.43 & 35.54 & 31.06 & 12.5 & 11.4 & 9.71 \\
\hline & Medium & 257.72 & 31.26 & 12.13 & & 9.9 & 8.27 \\
\hline & High & 515.26 & 31.50 & 6.11 & & 7.3 & 5.70 \\
\hline \multirow[t]{3}{*}{ НСТ (\%) } & Low & 17.33 & 1.95 & 11.22 & 3.0 & 2.8 & 1.18 \\
\hline & Medium & 36.57 & 3.46 & 9.46 & & 2.6 & 0.98 \\
\hline & High & 49.67 & 4.76 & 9.58 & & 2.5 & 0.85 \\
\hline \multirow[t]{3}{*}{$\operatorname{MCV}(f L)^{\dagger}$} & Low & 71.8 & 1.60 & 2.2 & 4.0 & 3.3 & 1.62 \\
\hline & Medium & 83 & 1.50 & 1.8 & & 3.2 & 1.52 \\
\hline & High & 92.7 & 2.08 & 2.2 & & 3.1 & 1.40 \\
\hline \multirow[t]{3}{*}{$\mathrm{MCH}(\mathrm{pg})^{\ddagger}$} & Low & 22.1 & 0.88 & 4.0 & 5.0 & 4.8 & 3.13 \\
\hline & Medium & 27.6 & 0.99 & 3.6 & & 4.7 & 3.08 \\
\hline & High & 31.1 & 1.19 & 3.8 & & 4.7 & 3.04 \\
\hline \multirow[t]{3}{*}{$\mathrm{MCHC}(\mathrm{g} / \mathrm{dL})^{\ddagger}$} & Low & 308.6 & 13.56 & 4.4 & 8.0 & 4.9 & 3.27 \\
\hline & Medium & 333 & 12.02 & 3.6 & & 4.7 & 3.01 \\
\hline & High & 337.1 & 12.64 & 3.8 & & 4.7 & 3.02 \\
\hline
\end{tabular}

${ }^{*}$ Lots LOTB0816L (low level), LOTB0816N (normal level) y LOTB0816H (high level). **The quality requirements came from the CLSI H26-A3 guide. ${ }^{\dagger}$ Indicates the quality objectives according to CLIA $88^{28}$. ${ }^{\ddagger}$ Ricos's Minimum analytical quality specifications ${ }^{22}$.

WBC: white blood cell, RBC: red blood cell, HB: hemoglobin, HCT: hematocrit, MCV: mean corpuscular volume, MCH: mean corpuscular hemoglobin, MCHC: mean corpuscular hemoglobin concentration, PLT: platelet

CLIA 88 indicates $(\mathrm{CV} \%) \pm 6 \%$ as a goal value of imprecision for the erythrocyte count (RBC) and HCT, $\pm 7 \%$ as target for the determination of $\mathrm{HB}, \pm 15 \%$ for the leukocyte count (WBC), and $\pm 25 \%$ as a goal for PLT count ${ }^{23}$. Our heterogeneous results showed that only WBC and HCT were within these parameters. Furthermore, that PLT and RBC surpassed the values indicated by CLIA at all levels (Table 2). These values indicated by CLIA are considered the most permissible when setting limits that are not as demanding, just as for HB that only exceeded the high-level limits.
When we compare our results with what was reported by Fink et al. ${ }^{18}$, we observe that HCT and HB exceeded the established limit value up to 3 times. On the other hand, WBC and RBC were within them. Similarly, a recent review by Vis and Huisman ${ }^{24}$ indicated different "state-of-the-art" acceptable limits of imprecision on reproducibility and inter-batches. All values of inaccuracy determined in this study disagree with these in large quantities at all levels of blood control, except $\mathrm{MCHC}$, which could not be compared and were able to 


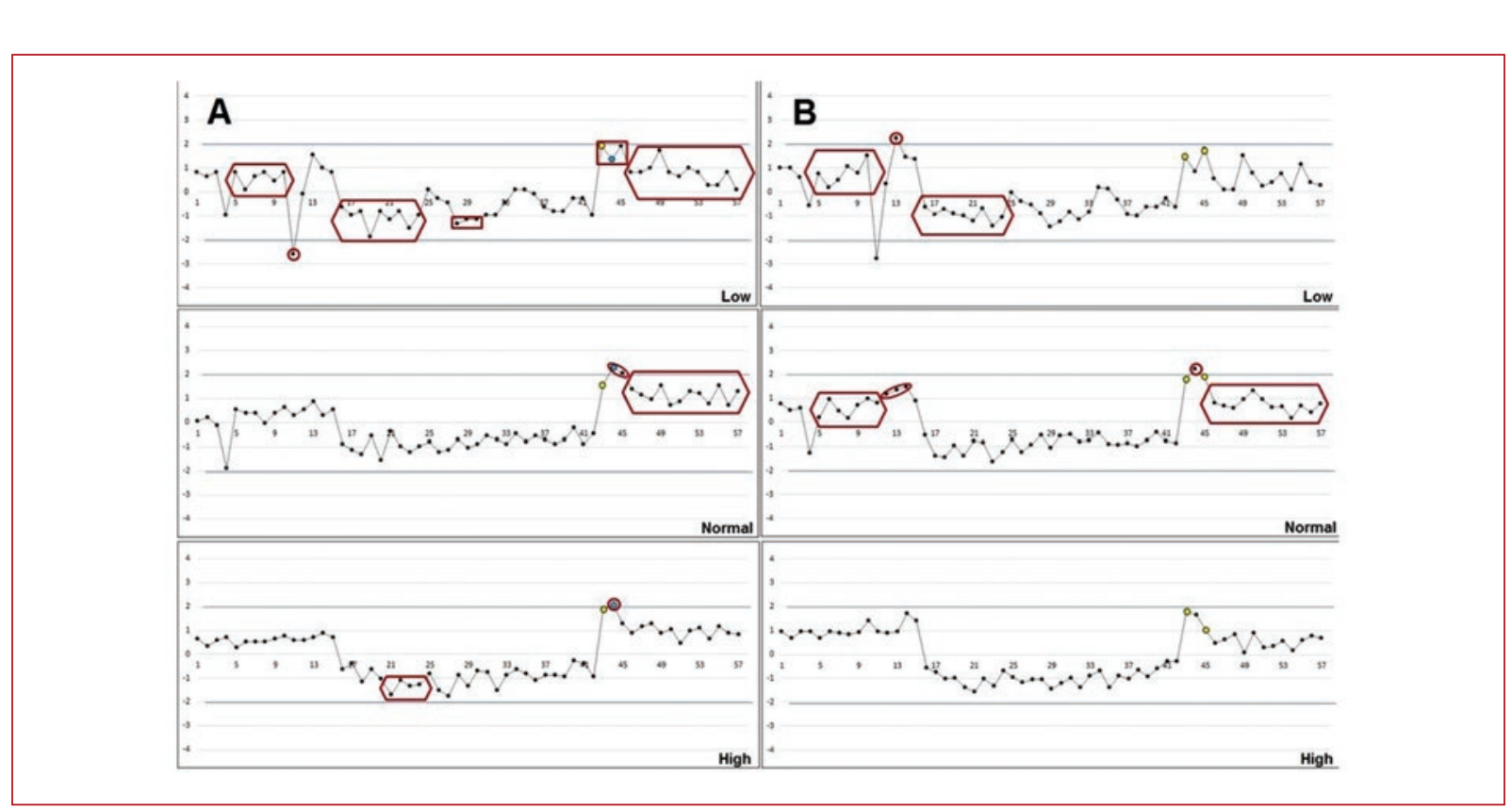

Figure 2. Levey-Jennings control chart of the daily runs of blood controls. The main infractions of the Westgard rules have been noted (red circles and squares). (A) Hemoglobin (g/dL), (B) hematocrit (\%). Lot: LOTB0816.

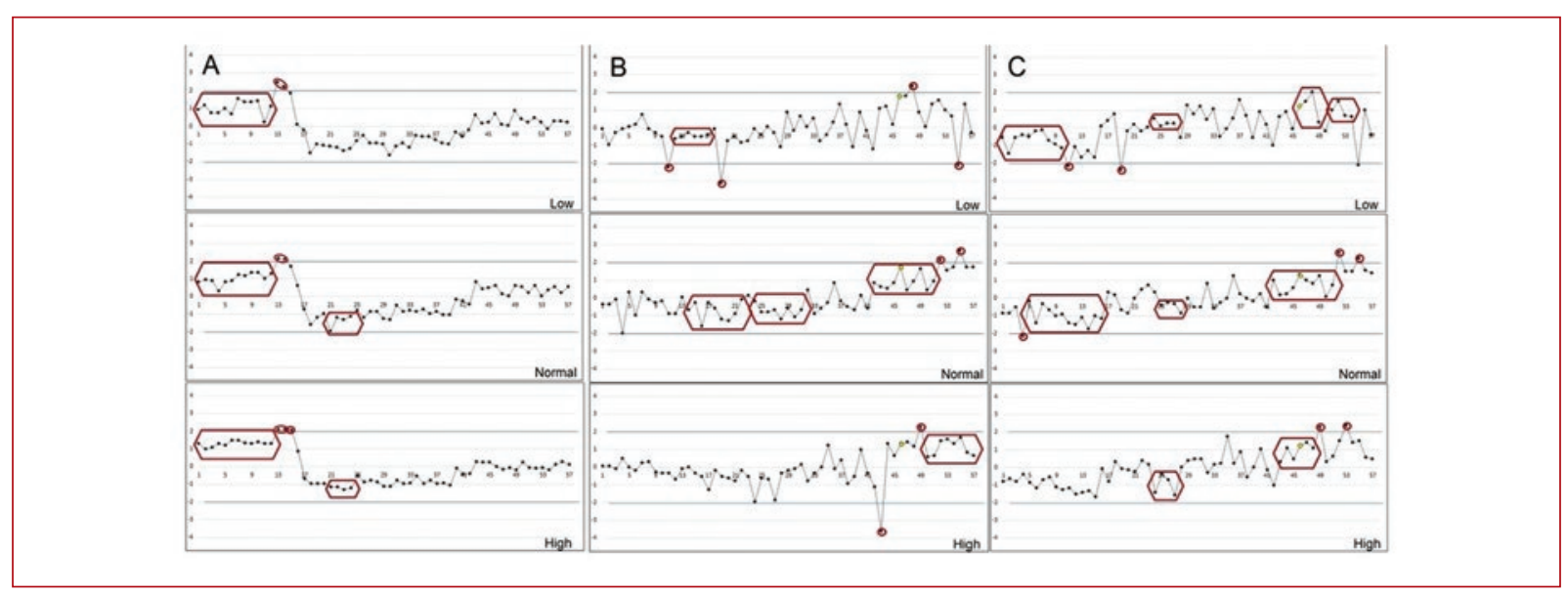

Figure 3. Levey-Jennings control chart of the daily runs of blood controls (hematimetric values). The main infractions of the Westgard rules have been noted (red circles and squares). (A) Mean corpuscular volume (fL), (B) mean corpuscular hemoglobin (pg), (C) mean corpuscular hemoglobin concentration (g/dL). Lot: LOTB0816.

affect the final result during the routine evaluation of patient samples.

The inaccurate results of the Auto hematology analyzer Landwind LW D3600 determined in this study when compared to the Samsung LABGEOHC10 hematology analyzer show that all parameters disagree between the imprecision values (WBC, $\mathrm{HB}, \mathrm{MCV}, \mathrm{MCH}$, and MCHCup to 4 times, RBC up to 8 times, and PLT up to 10 times more) in all levels of control ${ }^{25}$. When we compare the results of the evaluation of the Sysmex XE-2100D analyzer (except HCT) and the Sysmex XE2100 (except the erythrocyte indices), we observed that none of the hematological parameters showed agreement in the limits of imprecision determined in this study, evidencing a marked difference in performance between hematological analyzers ${ }^{26,27}$.

Furthermore, we observed differences in the bias for each parameter. The values for biases designated as 


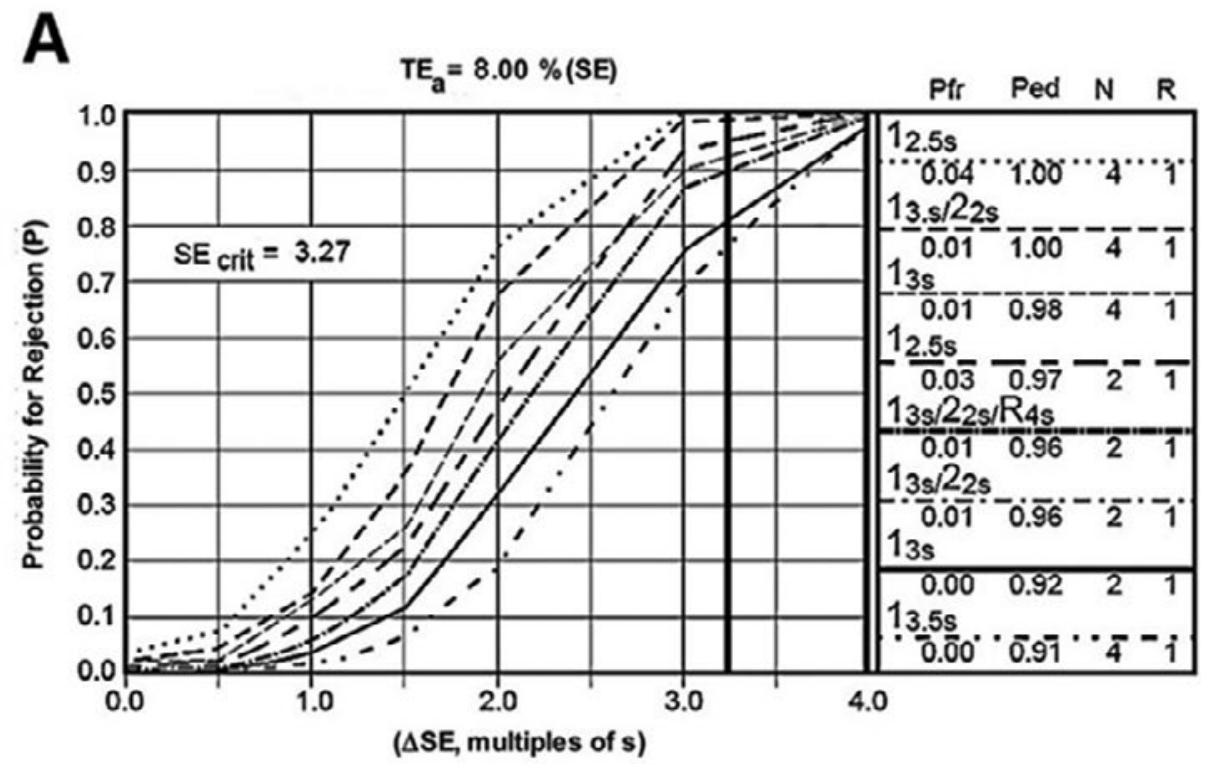

B

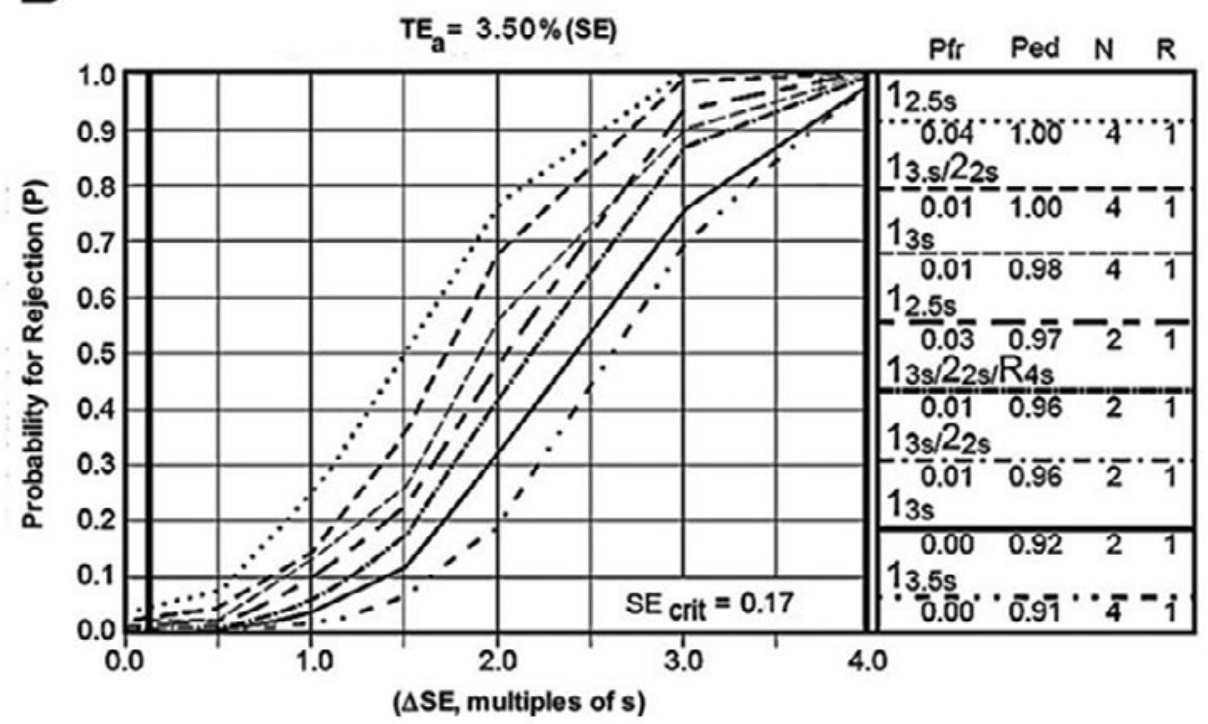

Figure 4. Power functions graphs for the statistical control of hematological parameters. In both cases, there are referred values of $\Delta S E$ opposites that imply the possibility of using Westgard control rules based on the probability of error detection $(\mathrm{Ped})$, and the probability of false rejection (Pfr) under a number $(\mathrm{N})$ of normalized controls. (A) Mean corpuscular hemoglobin concentrationin (g/dL) (low control). (B) Hemoglobin in ( $/ \mathrm{dL}$ ) (high control).

"desirable" by various authors ${ }^{28,29}$ show that only RBC and $\mathrm{WBC}$ are within range, that $\mathrm{MCV}, \mathrm{HCT}$, and $\mathrm{MCH}$ exceed the established range, and PLT alarmingly exceeded up to 6 times the desirable value of bias (desirable value: \pm 5.9 ).

In the Sysmex analyzers, the same phenomenon of discordance in the biases was seen in the vast majority of parameters. In fact, only WBC presented comparable values with the precision ranges of the Sysmex XE-2100D analyzer in the high and low control levels ${ }^{26,27}$. When contrasted with our results, other than $\mathrm{HB}$ and HCT, the comparison of the desirable limits of bias recently reported for the Sysmex XN hematological analyzer showed that only WBC and $\mathrm{MCH}$ were within these ranges in the three control levels ${ }^{30}$. Then as well, only our bias values for WBC 


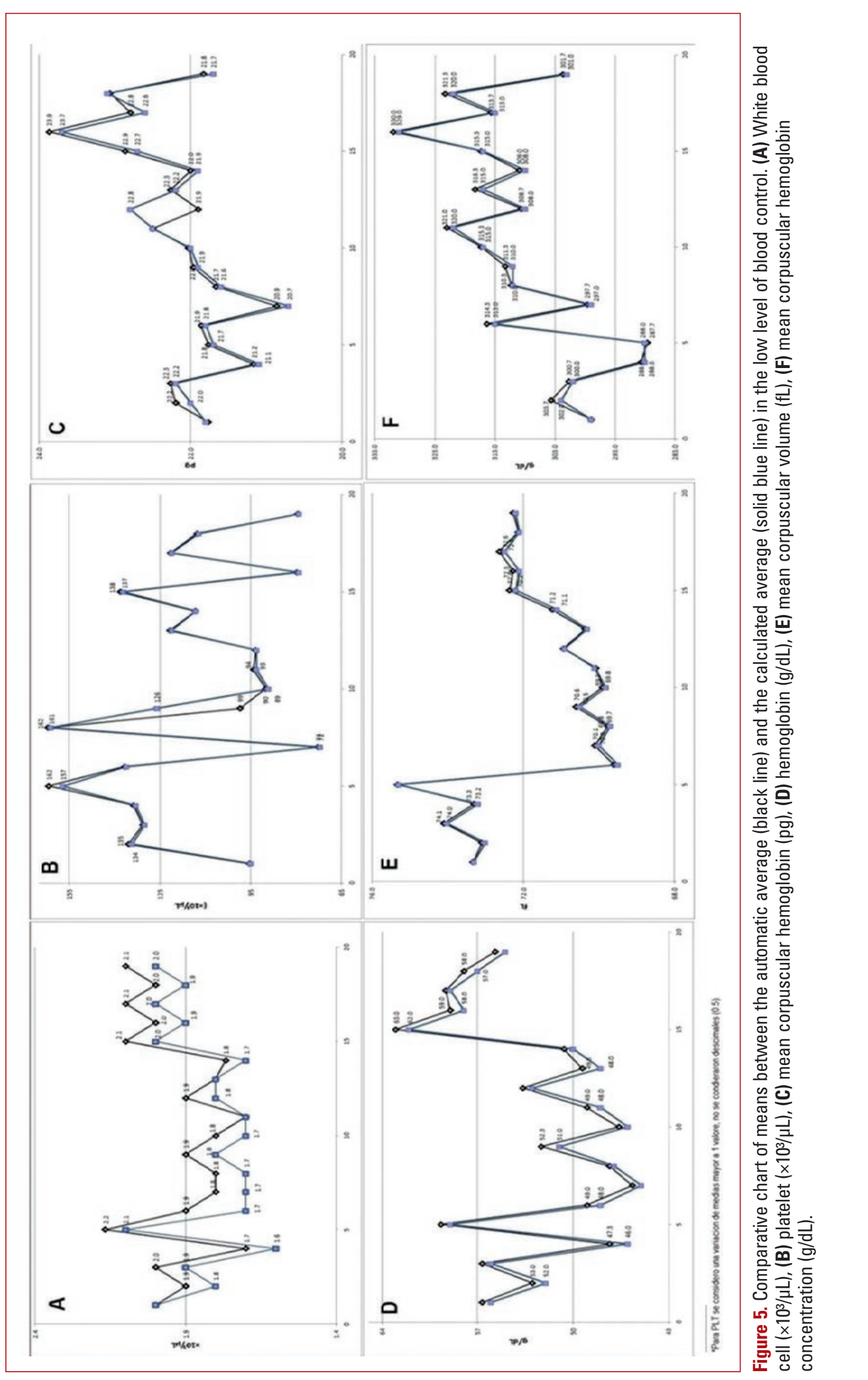


were within the range of optimal performance of other studies $^{29,31}$.

In the overall evaluation, we made with the Levey-Jennings control chart, we pointed out the $\geq 90$ infractions of the main Westgard rules in each control level, in addition to 7 violated multi-rules (Figs. 1-3). In general, the lower control presented more infractions to the rules (41 infractions) followed by the normal control (34 infractions) and the high (22). There were almost twice as many reports of systematic errors (64 infractions) than random reports (33 infractions).

The main erythrocytic components showed much inter-daily variability, usually by random $\left(1_{2 s}\right.$ y $\left.1_{3 s}\right)$ and systematic errors $\left(3_{1 \mathrm{~s}}, 6_{\mathrm{x}}, 9_{\mathrm{x}}\right.$, and $\left.2_{2 \mathrm{~s}}\right)$. Although in previous studies the stability of the erythrocyte indices ( $\mathrm{MCV}, \mathrm{MCH}$, and $\mathrm{MCHC}$ ) have been described in hematological analyses of patients ${ }^{32,33}$, in our study we observed 40 infractions of the Westgard rules, mainly of a random error type $\left(1_{2 s}\right)$. Similarly, for leukocytes and PLT, > 27 infractions were reported at all levels, mainly of the random error type $\left(1_{2 s}, y 1_{3 s}\right)$. In addition, trends were observed in different hematological parameters (more than five data gradually increased or decreased) (Figs. 1-3).

Corrective activities must have been implemented for all these infractions, providing solutions for the laboratory to carry on the daily activities of clinical analy$\mathrm{sis}^{7,11,14,15,20,34}$. Unfortunately, the real scene of the vast majority of laboratories in Peru, and surely in other countries, is different. Although new quality development objectives and sanitary provisions have been established for clinical laboratories, these are for various reasons (economic, technical, lucrative, etc.) not being adopted, or are being inappropriately implemented.

In this sense, clinical work is often disconnected with quality. Many laboratories do not use hematological controls, others use them monthly or annually, or wait until equipment is "calibrated" by the specialist technicians provided by the manufacturer. This problem can be explained by the lack of education about quality management, the costs involved in initiating quality circuits/processes, work overload, and the poor efficiency of health systems to meet the demand of personnel, along with the pursuit of profit.

These problems are reflected in the work flowchart of the laboratories. For example, according to different hematology laboratory guidelines, only in cases of suspected hematological alteration should a direct review of peripheral blood smears and manual differential leukocyte counts be performed. However, this is carried out in each evaluation (whether the hematological alteration is present or not), leading to unnecessary expenses, delays in the emission of results, and inefficiency of man-hour costs due to the low quality of results of these health-care centers. This happens not only in the laboratories of low strata but also in those of great prestige and high specialization ${ }^{35}$.

For more than three decades now, the evaluation of organizational performance has been improved with the sigma metric, eliminating defects, and errors ${ }^{36}$. At present, its use in clinical analysis laboratories and in health care not only ensures reliable and efficient results but also allows the fulfillment of competencies and continuous improvement, in addition to international certification and accreditation.

These should be a priority for health systems of all countries, systems that allow education and training of staff to develop these processes in their work and with the tools they have. However, the National Institute of Health of Peru, as an example of these cases, does not refer to any recommendation on the processes of quality management in hematology, neither for manual processes nor for automated ones ${ }^{37,38}$.

In this study, we determined sigmas and $\triangle S E$ to be heterogeneous between hematological parameters and control levels (Table 2); the performance characteristics were optimal for WBC and PLT $(\Delta S E=\geq 5.8)$. They were also deficient for all erythrocyte parameters $(\Delta \mathrm{SE}=$ 0.17-3.27).

The power functions graphs describe the acceptable errors and inaccuracies and the quality control that is necessary to monitor the performance of the method (just like the OPSpecs or sigma charts). These control charts also evaluate the performance of the test under stable conditions and warn of changes that may affect the report. Their objective is to obtain $\leq 5 \%$ probability of false rejection and $\geq 90 \%$ Ped in a single analytical run $^{10,17,34}$.

The main erythrocyte components showed low $\Delta \mathrm{SE}$ and significative daily variability. Although WBC obtained a high performance (sigma and $\triangle \mathrm{SE}$ ), it presented 31 infractions of the Westgard rule at all levels, as well as 37 infractions in PLT. If the institution had done quality planning, it would have established the rules and/or multi-rules necessary for the control of the analyses. Some of these infractions would have been omitted because they were considered part of the analytical run, and others that indicate systematic or random errors, which affect the results, would be corrected ${ }^{10,17,20}$.

In addition to these important evaluations of performance and quality in the hematology laboratory, the American College of Pathologists indicates that the use 


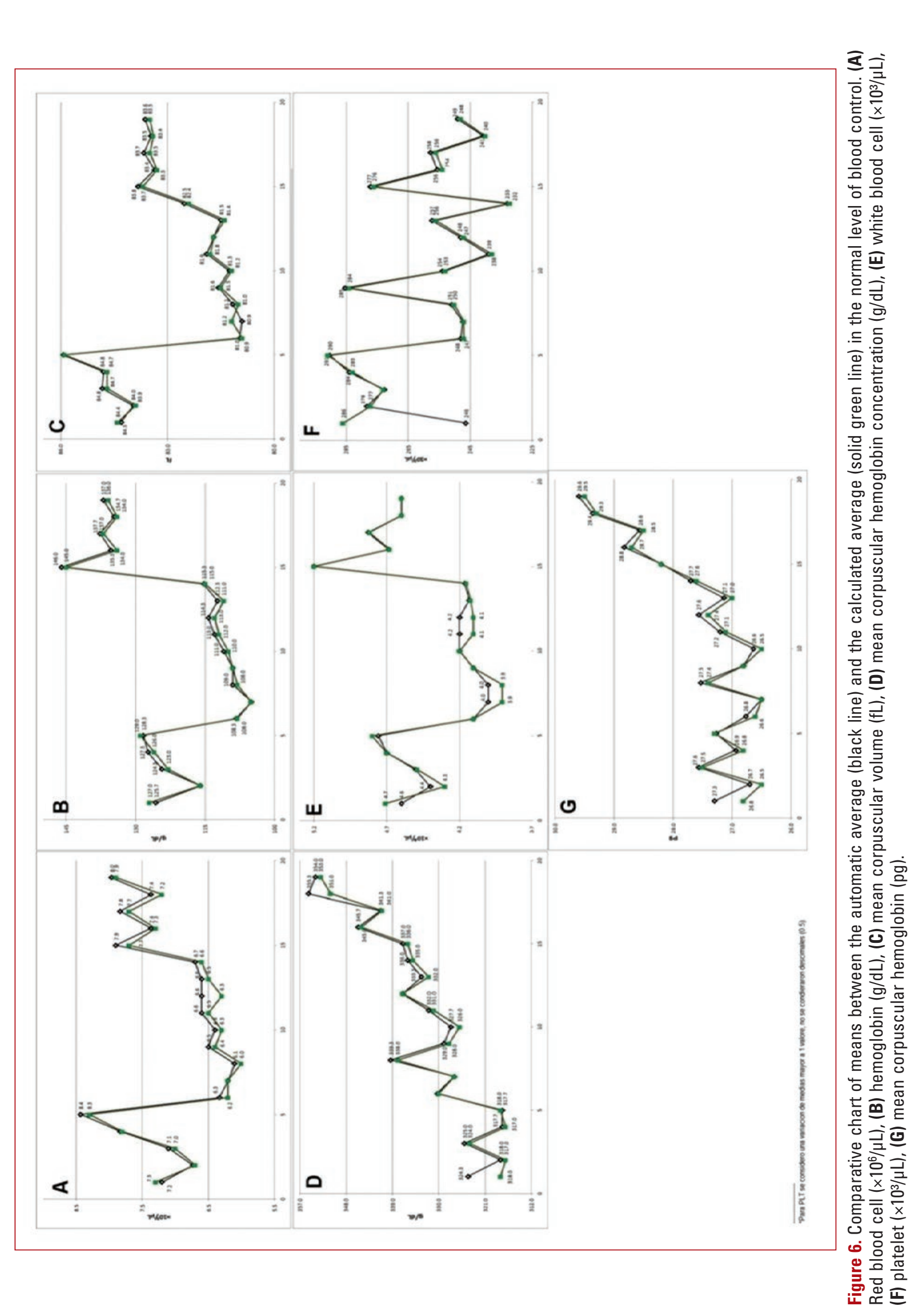







of controls with stabilizers, specimens of retained patients, or a mixture of these should be included, to act as monitors of any changes in the means of the hematological parameters ${ }^{39}$. All these variables depend on the sample and control storage hours, the type of storage, their rational use, and the established guidelines, as well as the time and type of mixture before use and the number of determinations, as well as their calculation, etc. ${ }^{29,40}$.

Regarding the latter, we reported inconsistencies in the establishment of means for each hematological parameter, finding differences between daily results and among levels of control. We identified significant differences between the determined automatic mean (by the autoanalyzer) and the calculated mean, having in many cases marked differences (Figures 5-7). RBC showed greater robustness and agreement in the estimation of means, in general, in the three levels of controls.

The HCT and RBC (56 and 46 results, respectively) were those with the highest agreement of means, followed by PLT (17 results), MCV (13 results), and MCH (9 results). The very rigorous evaluation character (decimal evaluation in $\mathrm{g} / \mathrm{dL}$ ), could explain why $\mathrm{MCHC}$ (5 agreements) obtained low agreements, but worryingly, does not explain those obtained for HB (6 agreements). These 142 agreements of means (calculated and automatic) allowed us to establish these as the daily values (after 3 analyses of the same serial control), in addition to those used to evaluate the quality of the autoanalyzer and the daily results ${ }^{11}$. Regardless of the deviation values of the manufacturer that situate the majority of averages within these parameters, in this analysis, we demonstrate the variability between the determination of means by the equipment (in the measurement of three runs of each control for the estimation of the average mean) compared to that determined by the user (such as by checking daily averages).

Our results should be interpreted considering the following two limitations. First, that the evaluation was not performed with the new CLSI EP15-A3 guide for the conditions and access of the laboratory to this information (a study that should be carried out given that this new guide presents substantial modifications of the quality assessment processes). Second, the elimination of extreme values was not performed with the Dixon or Grubbs test ${ }^{41}$.

Finally, it has been recommended that each laboratory should estimate their quality control ranges for each type of analyzer based on the recommendations of several guidelines ${ }^{20,42-44}$. However, these regulations are still not very accessible or well-known by the workers at the hematological laboratory (technicians, phlebotomists, medical technologists, medical service managers, and manufacturers), or failing that; their use is dispensed with because it implies, among other things, investment in quality.

In Peru, both the Peruvian Institute of Quality of Peru (INACAL) and the National Institute of Health and the Peruvian Association of Quality Management of Peru (ASPEGC) must issue regulations and guidelines that allow clinical laboratories to manage the quality processes, as has been established in other countries ${ }^{14}$. We hope that this will happen in the coming years in Peru, and throughout the region.

\section{Conclusions}

The quality evaluation under the $\Delta S E$ analysis showed a heterogeneous performance of eight hematological parameters and between the intra-parameter control levels in the Landwind LW D3600 analyzer. We also determined $>90$ infractions of the Westgard rules and mismatches in the determination of means (calculated and automatic) in 1368 trials in Lima, Peru.

Although the controls were outside the limits of one or more parameters, patient samples were analyzed, threatening health security and generating uncertain results. These could have medically important errors that affect the patient's health.

\section{Ethical disclosures}

Protection of humans and animals. The authors declare that the followed procedures conform with the ethical guidelines of the human committee of responsible human experimentation and in accordance with the World Medical Association and the Helsinki Declaration.

Data confidentiality. The authors declare that they have followed the protocols of their center about publishing patient data.

Right to privacy and informed consent. The authors have obtained the informed consent of the patients and/or of all the subjects referred in this articles. This document is in the hands of the corresponding author.

\section{Funding}

Self-financed by the authors. 


\section{Conflict of interest}

The authors declare that they have no financial or labor conflicts of interest.

\section{References}

1. Glaser AK, Reder NP, Chen Y, et al. Light-sheet microscopy for slide-free nondestructive pathology of large clinical specimens. Nat Biomed Eng. 2017:1:84

2. Moya-Salazar J, Bruno M, Rojas-Zumaran V, Bhamla S, Prakash M Implementation of the Foldscope as a device for cervical cancer diagnosis: a verification and evaluation study. Rev Latinoamer Patol Clin. 2016;63:141-7.

3. Bhamla MS, Benson B, Chai C, Katsikis G, Johri A, Prakash M Hand-powered ultralow-cost paper centrifuge. Nat Biomed Eng. 2017:1:9.

4. Moya-Salazar J, Rojas-Zumaran V. Eco-Pap: the ecological modification of Papanicolaou stain for sustainable cervical cancer diagnosis. Acta Cytol. 2018, in press.

5. Zhang H, Qiu X, Zou Y, et al. A dye-assisted paper-based point-of-care assay for fast and reliable blood grouping. Sci Transl Med. 2017;9.

6. Kohn LT, Corrigan JM, Donaldson MS. Committee on Quality of Healthcare in America, Institute of Medicine. To Err is Human: building a Safer Health System. Washington DC, USA: National Academy Press; 2000.

7. Moya-Salazar J, Pio-Dávila L, Diaz RM. Depletion of blood supply and cost for indeterminate donations at Hospital Nacional Guillermo Almenara Irigoyen. Horiz Med. 2017;17:31-7.

8. Badrik T. Quality leadership and quality control. Clin Biochem Rev. 2003;24:81-93.

9. Terrés-Speziales A. Six sigma: determinación de metas analíticas con base en la variabilidad biológica y la evolución tecnológica. Rev Mex Patol Clín. 2007:54:28-39.

10. Westgard JO, Groth T. Power functions for statistical control rules. Clin Chem. 1979;25:863-9.

11. Moya-Salazar J, Pio-Dávila L. Day-per-day maintenance, six-sigma and analytic quality in hematology: quality verification of hematological auto-analyzer Landwind LW D3600 in Peruvian health-care center level II. Rivista Italiana della Medicina di Laboratorio 2018; in press.

12. Westgard JO. Six Sigma Quality Design and Control Detail. Madison: Westgard Inc.; 2001.

13. Uldall A. Quality assurance in clinical chemistry. Scand J Clin Lab Invest. 1987;47 Suppl 187:1-95

14. Gómez LR, Moscoso EH, Retamales CE, Valenzuela BC. Guía Técnica Para Control de Calidad de Mediciones Cuantitativas en el Laboratorio Clínico. Santiago: Departamento Laboratorio Biomédico Nacional y de Referencia. Ministerio de Salud Chile; 2015

15. Cembrowski GS, Smith B, Tung D. Rationale for using insensitive quality control rules for today's hematology analyzers. Int J Lab Hematol. 2010 32:606-15.

16. Cembrowski GS, Westgard JO. Quality control of multichannel hematology analyzers: evaluation of bull's algorith. Am J Clin Pathol. 1985;83: 337-45.

17. Moya-Salazar J, Pio-Davila L. Evaluation of inter-batch variability in establishing and quality control of glucose. Med Univ. 2016;18:85-90.

18. Fink NE, Fernández AF, Mazziotta D. Evaluación externa de la calidad analítica en hematología: una necesidad en América Latina. Pan Am J Public Health 1997:2:181-8.

19. Clinical and Laboratory Standards Institute (CLSI). User Verification of Performance for Precision and Trueness. Approved Guideline. $2^{\text {nd }}$ ed. CLSI Document EP-15-A2. Wayne, Pennsylvania, USA: Clinical and Laboratory Standards Institute; 2006.

20. Clinical and Laboratory Standards Institute (CLSI).Validation, Verification and Quality Assurance of Automated Hematology Analyzers. $2^{\text {nd }}$ ed. Wayne, Pennsylvania, USA: CLSI document H26-A2. Vol. 30. Clinical and Laboratory Standards Institute; 2010.

21. Nikolac N, Supak-Smolcić V, Simundić AM, Celap I, Croatian Society of
Medical Biochemistry and Laboratory Medicine. Croatian society of medical biochemistry and laboratory medicine: national recommendations for venous blood sampling. Biochem Med (Zagreb). 2013;23:242-54.

22. Ricós $\mathrm{C}$, Ramón $\mathrm{F}$, Salas A, et al. Interdisciplinary expert committee fo quality specifications in clinical laboratory. Minimum analytical quality specifications of inter-laboratory comparisons: agreement among Spanish EQAP organizers. Clin Chem Lab Med. 2011;50:455-61.

23. Clinical Laboratory Improvement. Amendments. . CLIA proficiency testing criteria for acceptable analytical performance. Federal Reg. 1992;57:7002-186.

24. Vis JY, Huisman A. Verification and quality control of routine hematology analyzers. Int J Lab Hematol. 2016;38 Suppl 1:100-9.

25. Park IJ, Ahn S, Kim Yl, Kang SJ, Cho SR. Performance evaluation of samsung LABGEO(HC10) hematology analyzer. Arch Pathol Lab Med. 2014;138:1077-82.

26. Santos TE, Comar SR, Beltrame MP. Performance evaluation of the Sysmex® XE-2100D automated hematology analyzer. J Bras Patol Med Lab. 2014;50:26-35.

27. Walters J, Garrity P. Performance evaluation of the Sysmex XE-2100 hematology analyzer. Lab Hematol. 2000;6:83-92.

28. Ricos C, Alvarez V, Cava F, et al. Current databases on biologic variation: pros, cons and progress. Scand J Clin Lab Investig. 1999;59:491-500.

29. Pintér E, László K, Schüszler I, Konderák J. The stability of quantitative blood count parameters using the ADVIA 2120i hematology analyzer. Pract Lab Med. 2016;4:16-21.

30. Daves M, Zagler EM, Cemin R, et al. Sample stability for complete blood cell count using the sysmex XN haematological analyser. Blood Transfus. 2015;13:576-82.

31. Buoro S, Mecca T, Seghezzi M, et al. Assessment of blood sample stability for complete blood count using the sysmex XN-9000 and mindray BC-6800 analyzers. Rev Bras Hematol Hemoter. 2016;38:225-39.

32. Koepke JA, Protextor TJ. Quality assurance for multichannel hematology instruments. four year experiences with patient mean erythrocyte indices. Am J Clin Path. 1981;75:28.

33. Bull BS, Korpman RE. Intralaboratory quality control using patient data. In: Cavill I, editor. Methods in Hematology Quality Control. New York: Churchill Livingston; 1982.

34. Westgard JO. Quality control how labs can apply six sigma principles to quality control planning. Clin Lab News. 2006;32:10-2.

35. Bain BJ. Diagnosis from the blood smear. N Engl J Med. 2005:353:498-507.

36. Barry R, Murcko AC, Brubaker CE. The Six Sigma Book for Healthcare, Improving Outcomes by Reducing Errors. Chicago: Health Administration Press, ACHE Management Series; 2002.

37. Muñoz ZM, Morón CC. Manual de procedimientos de laboratorio en técnicas básicas de hematología. Lima: Ministerio de Salud del Perú. Instituto Nacional de Salud; 2005

38. Zurita MS. Manual Procedimientos de Laboratorio, Laboratorios Locales I, Laboratorios Locales II. Lima: Ministerio de Salud del Perú. Instituto Nacional de Salud Perú; 2013.

39. College of American Pathologists (CAP). Hematology and Coagulation Checklist. HEM.25870 Commercially Assayed Controls. Northfield; IL: College of American Pathologists; 2012.

40. Hill VL, Simpson VZ, Higgins JM, et al. Evaluation of the performance of the sysmex XT-2000i hematology analyzer with whole bloods stored at room temperature. Lab Med. 2009;40:709-18.

41. Clinical and Laboratory Standards Institute (CLSI). Defining, Establishing, and Verifying Reference Intervals in the Clinical Laboratory-Approved Guideline. CLSI document C28-A3. Wayne, Pennsylvania, USA: Clinical and Laboratory Standards Institute; 2008.

42. Clinical and Laboratory Standards Institute (CLSI). Statisticat Quality Control for Quantitative Measurement Procedures: principles and Definitions: approved Guideline. CLSI document C24-A3. Wayne, Pennsylvania, USA: Clinical and Laboratory Standards Institute; 2006.

43. Clinical and Laboratory Standards Institute (CLSI). Laboratory Quality Control Based on Risk Management Approval Guideline. CLSI document EP23-A. Wayne, Pennsylvania, USA: Clinical and Laboratory Standards Institute; 2011.

44. International Organization for Standardization (ISO). Medical Laboratories - Particular requirements for quality and competence. ISO 15189. Geneva, Switzerland: International Organization for Standardization; 2007. 